\title{
Dissociation between priming and recognition in the expression of sequential knowledge
}

\author{
DAVID R. SHANKS \\ University College London, London, England \\ and \\ PIERRE PERRUCHET \\ Université de Bourgogne, Dijon, France
}

\begin{abstract}
Exposure to a repeating sequence of target stimuli in a speeded localization task can support both priming of sequence-consistent responses and recognition of sequence components. Here, a task is introduced in which measures of priming and recognition are obtained concurrently, and it is demonstrated that priming of sequence-consistent responses occurs even when test stimuli are not recognized. The results show that sequence knowledge can be expressed in the absence of conscious recognition. However, we also show that this result is consistent with a simple model in which priming and recognition depend on exactly the same underlying memory strength variable.
\end{abstract}

Exposure to a repeating sequence of target stimuli in a speeded localization task can support both priming of sequence-consistentresponses and recognition of sequence components (Fendrich, Healy, \& Bourne, 1991; Marsolek \& Field, 1999; Perruchet \& Amorim, 1992; Shanks \& Johnstone, 1999; Willingham, Greeley, \& Bardone, 1993). Priming is an example of implicit memory and is often argued to be independent of explicit memory, which supports recognition (N. J. Cohen \& Eichenbaum, 1993; Gabrieli, 1998; Schacter \& Buckner, 1998). One widely employed task for studying sequence learning is a perceptual-motor localization task originally devised by Nissen and Bullemer (1987) and subsequently adopted and modified by many others (Cleeremans \& McClelland, 1991; Destrebecqz \& Cleeremans, 2001; Frensch, Lin, \& Buchner, 1998; Honda et al., 1998; Jiménez, Méndez, \& Cleeremans, 1996; Perruchet \& Amorim, 1992; Perruchet, Bigand, \& Benoit-Gonin, 1997; Reber \& Squire, 1998; Reed \& Johnson, 1994; Shanks \& Johnstone, 1999; Stadler, 1995; Willingham et al., 1993). Participants perform a choice reaction time (RT) task in which a target stimulus appears at one of a small number (e.g., four) of locations, with the successive locations being determined by an underlying sequence. Participants' sequence knowledge may be expressed either indirectly, via reduction in response latency to predictable targets (priming), or directly, via recall, recognition, prediction, or generation tests thought to require conscious knowledge.

This research was supported by the United Kingdom Economic and Social Research Council and by the Leverhulme Trust. We thank Bob Boakes, Axel Cleeremans, Arnaud Destrebecqz, Richard Tunney, and Mike Stadler for their helpful comments. Correspondence should be addressed to D. R. Shanks, Department of Psychology, University College London, Gower Street, London WC1E6BT, England (e-mail: d.shanks@ ucl.ac.uk).
Although it has been repeatedly claimed that direct and indirect measures of sequence knowledge can be dissociated, previous results have in fact been highly equivocal. Key results appearing to demonstrate dissociations have not been replicated (see Curran, 1997; Shanks \& Johnstone, 1999) or have been criticized (Dienes \& Berry, 1997; Perruchet \& Amorim, 1992; Perruchet \& Gallego, 1993; Perruchet, Gallego, \& Savy, 1990; Shanks \& Johnstone, 1998; Shanks \& St. John, 1994) on a variety of methodological grounds. To take just one example particularly pertinent to the present work, it is almost always the case that the direct and indirect measures are taken at different times in distinct test phases. This creates a number of potential difficulties: For instance, if the direct test is administered some time after the indirect test, the results may be differentially affected by forgetting. Moreover, with distinct test phases, participants may be inclined to adopt different response sets, response biases, strategies, levels of motivation, and so on, which may significantly affect the relative levels of performance obtained in the tests. The availability of a testing method that enables concurrent direct and indirect knowledge assessment would allow many methodological problems of this sort to be finessed.

In the present study, such a method is developed and exploited. Sequence learning was induced by presenting participants with a 12-element sequence of targets (e.g., 1-2-13-4-2-3-1-4-3-2-4), in which 1-4 refer to locations on a computer screen. In these second-order conditional sequences (Reed \& Johnson, 1994), two elements of context (prior locations) are required to fully predict the next target. The sequence cycled continuously without interruption. Participants responded to each target by pressing as fast as possible a corresponding key on the keyboard, and their response latencies were measured. In the first experiment, training comprised 6,10 , or 14 blocks of 100 trials, whereas 
in the second experiment, there were 17 blocks. After a break, 24 test trials were administered, which comprised short sequences of three (Experiment 1) or six (Experiment 2) targets. Twelve of these sequences were extracted from the training sequence, and 12 were inconsistent with the training sequence. Participants responded as rapidly as possible to the targets as in the training stage and then made a recognition rating on a 6-point scale.

Importantly, recognition was measured concurrently with priming (the tendency for old test sequences to be executed faster than new ones). The key questions are, first, whether any dissociation can be detected between these direct and indirect measures of sequence knowledge under these conditions. This question is addressed by computing the relative execution speeds for old and new test trials for which participants give identical recognition ratings. If, for example, priming and recognition are dependent on distinct knowledge sources, priming might be expected even when old and new sequences are not differentially recognized. Alternatively, if priming and recognition are tightly coupled (Shanks \& Johnstone, 1999), old and new test sequences given identical recognition ratings should be executed with equivalent latencies. The second question is whether a dissociation, if found, requires the postulation of separate memory stores or, alternatively, is compatible with a model in which priming and recognition depend on exactly the same underlying memory strength variable.

\section{METHOD}

\section{Training Phase}

Since the two experiments reported here differed in only a few procedural details, they are described together. The present Experiment 2 is the same as Experiment 3 of Shanks and Johnstone (1999): Analyses are reported here that were not conducted by Shanks and Johnstone.

\section{Participants}

The participants ( $n=69$ and 79 in Experiments 1 and 2, respectively) were healthy, young adults naive to the purpose of the experiment. Twenty-six, 21, and 22 participants were assigned to groups receiving 6, 10, or 14 training blocks, respectively, in Experiment 1.

The experiments were fully automated. Stimulus presentation, RT measurement, and response recording were all controlled by computer. Four boxes were arranged horizontally at the bottom of the computer screen, drawn with white lines against a blue background. The boxes were $3.5 \mathrm{~cm}$ wide and $2 \mathrm{~cm}$ high. A dot $(2 \mathrm{~mm}$ in diameter) appeared in the center of one of these boxes on each target-location trial. Target locations were enumerated as $1-4$, from left to right. The participants were instructed to indicate locations $1-4$ as quickly as possible by using the V, B, N, and M keys located across the bottom of the keyboard, respectively. They responded to locations 1 and 2 with the middle and index fingers of their left hands, respectively, and to locations 3 and 4 with the index and middle fingers of their right hands, respectively.

Each block of 100 target-location trials began at a random point in the sequence, and, thereafter, targets appeared according to the sequence that corresponded to the particular condition and block type. A target-location trial ended when a participant pressed the correct key, at which time the target was erased. The next trial began $200 \mathrm{msec}$ later. Response latencies were measured from the onset of the targets to the completion of correct responses. There was a break after every block of trials. Two different second-ord er conditional (Reed \& Johnson, 1994) sequences (S1: 1-2-1-3-4-2- 3-1-4-3-2-4 and S2: $1-2-3-4-1-3-2-1-4-2-4-3)$ were used, and approximately half the participants in each group and experiment received each sequence. Data are collapsed across these counterbalancing subgroups. In Experiment 1 , the participants received 6,10 , or 14 training blocks. Following standard practice (Stadler, 1995), in the second to last block, the alternate sequence was used. ${ }^{1}$ In Experiment 2, there were 17 training blocks, and the transfer sequence was used on block 15 .

Concurrently with the target-location task, the participants performed a tone-counting task (A. Cohen, Ivry, \& Keele, 1990) to divert their attention away from the structured sequence of targets, hence, minimizing the involvement of explicit sequence learning. A 100-msec computer-generated tone was emitted 100-msec after each correct target-location response. Each tone was randomly determined to be either low $(1000 \mathrm{~Hz})$ or high $(2000 \mathrm{~Hz})$, and the participants were instructed to count the number of high tones emitted during each block of trials. At the end of each block, the participants were asked to provide their count. If they made less than 5\% errors, they were informed that their tone counting was accurate and were asked to continue their good performance. If they made $5 \%$ or more errors, they were told their error percentage and were encouraged to try harder to attend to their tone-counting accuracy.

\section{Recognition and Priming Test}

After the final training block, the participants were informed about the sequence and were asked to perform a recognition test. There were 24 test sequences in total, presented in a randomized order for each participant. The major difference between the experiments was that sequences comprised three elements in Experiment 1, but six in Experiment 2. Twelve of the sequences were constructed by starting at each serial location of S1, and 12 were constructed by starting at each serial location of S2. Thus the S1 test sequences were old for participants trained on $\mathrm{S} 1$ and new for participants trained on $\mathrm{S} 2$, and vice versa for the S2 sequences, though it should be noted that the terms old and new are relative: In fact, all test sequences had been seen before, with the new sequences having been part of the transfer sequence. Both ratings and trial-by-trial response latencies were recorded for the test sequences. Ratings were made on a scale of 1 to 6 , where $6=$ certain I have seen this sequence before, $5=$ fairly certain I have seen this sequence before, $4=$ guess I have seen this sequence before, $3=$ guess $I$ have not seen this sequence before, $2=$ fairly certain I have not seen this sequence before, and $1=$ certain I have not seen this sequence before. In Experiment 2, the rating scale was reversed, but for consistency with Experiment 1, these scale values are reversed in the following analyses.

\section{Data Analysis}

Individual trials on which RTs were greater than $1,000 \mathrm{msec}$ were excluded from all analyses of test trial response latencies $(3.2 \%$ and $2.1 \%$ of trials in Experiments 1 and 2, respectively). Because the participants did not always use every recognition category for both old and new test sequences, the data presented in Figure 3 below only include the participants who used the given category on at least one old and one new test trial.

\section{RESULTS}

Neither priming nor recognition varied in Experiment 1 as a function of number of training blocks, and therefore data were collapsed across this factor. A recognition score for each participant was computed as the difference between the mean judgment for old sequences minus the mean judgment for new sequences. Figure 1 shows that abovechance recognition was evident in both experiments [Ex- 

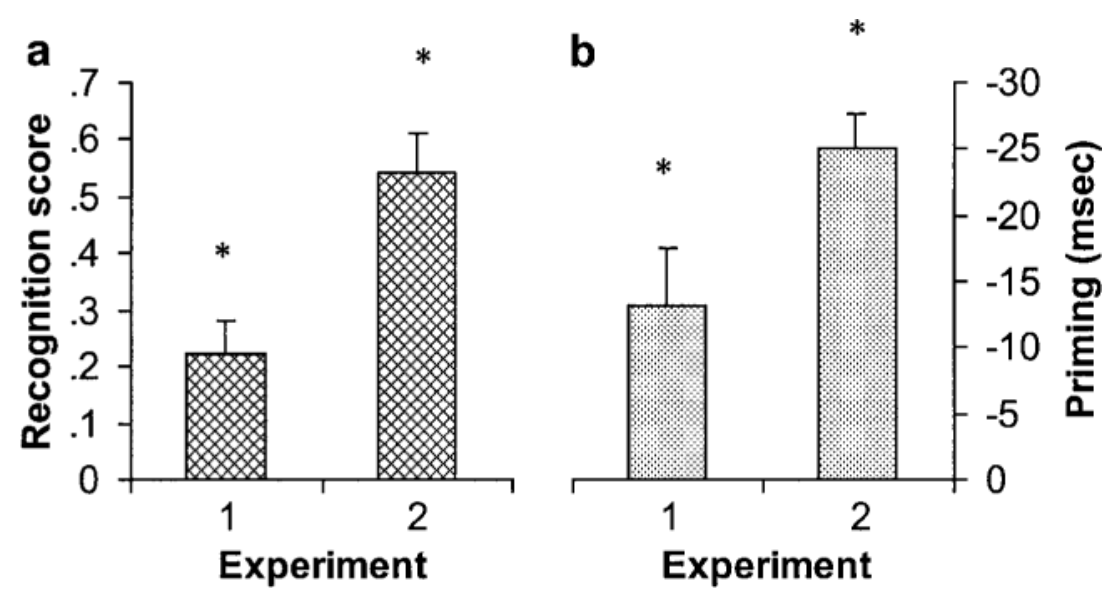

\begin{abstract}
Figure 1. (a) Mean (+SEM) recognition scores in Experiments 1 and 2. Participants responded to 12 old and to 12 new sequences and then made a recognition judgment $(1=$ certain new, $6=$ certain old $)$. Sequences comprised three (Experiment 1$)$ or six (Experiment 2) elements. (b) Mean (+ $S E M$ ) priming scores across the same test trials. Asterisks indicate that these results differ from chance (zero), with $p<.05$.
\end{abstract}

periment $1, t(68)=3.66, p<.001 ;$ Experiment $2, t(78)=$ $7.54, p<.001]$, but was significantly greater in the latter $[t(144.7)=3.39, p<.001](d f$ adjusted according to Levene's test). ${ }^{2}$

Figure 1 also presents the mean priming scores. For Experiment 1 , these were computed by taking the difference between RTs to the third target of each old sequence and the comparable RTs for each new sequence; priming was not expected for the first and second targets because two elements of context were required in order to discriminate old from new sequences. For Experiment 2, priming scores were based on mean RTs to Targets 3-6 of each test sequence. Negative priming scores indicate faster responding to the old sequences and hence retrieval of sequence knowledge. Above-chance priming occurred in both experiments [Experiment $1, t(68)=3.06, p<.005$; Experiment $2, t(78)=9.52, p<.001]$. However, priming was again greater in Experiment $2[t(114.8)=2.38, p<.02](d f$ adjusted according to Levene's test). Supporting the idea that six-element test sequences should be easier to discriminate than three-element ones, both priming and recognition scores were very much larger with the longer test sequences.

Figure 2 illustrates the priming effect more directly. The figure shows mean RTs to Targets 1-3 (Experiment 1) and 1-6 (Experiment 2) for old and new test sequences. Consistent with the idea that, with second-order conditional sequences, predictive knowledge can only be accessed for the third and subsequent targets for old versus new sequences, the figure reveals that RTs were comparable for the first and second target of each test sequence but diverged for the third, predictable, target by about $17 \mathrm{msec}$. In Experiment 2, RTs diverged for each of the four predictabletargets (3-6). A two-way repeated measures analysis of variance (ANOVA) on the Experiment 1 data revealed no overall effect of the old/new variable $[F(1,68)=1.48, p>.2]$, a reliable effect of target $[F(2,136)=432.03, p<.001]$, and a reliable interaction $[F(2,136)=3.07, p<.05]$. Paired comparisons revealed that RTs for old versus new sequences did not differ on the first and second targets $[t(68)<1, p>.3$, in each case], but those for the third target did $[t(68)=3.18$, $p<.005]$. A similar ANOVA on the Experiment 2 data revealed an overall effect of the old/new variable $[F(1,78)=$ $69.36, p<.001]$, a reliable effect of target $[F(5,390)=$ $138.14, p<.001]$, and a reliable interaction $[F(5,390)=$ $10.46, p<.001]$. Paired comparisons revealed that RTs for old versus new sequences did not differ on the first or second targets $[t(78)<1.15, p>.2$, in each case], but those for the subsequent targets did $[t(78)>5.20, p<.001$, in each case].

The critical data concern relative RTs for old and new test sequences, given identical recognition ratings. Fig-

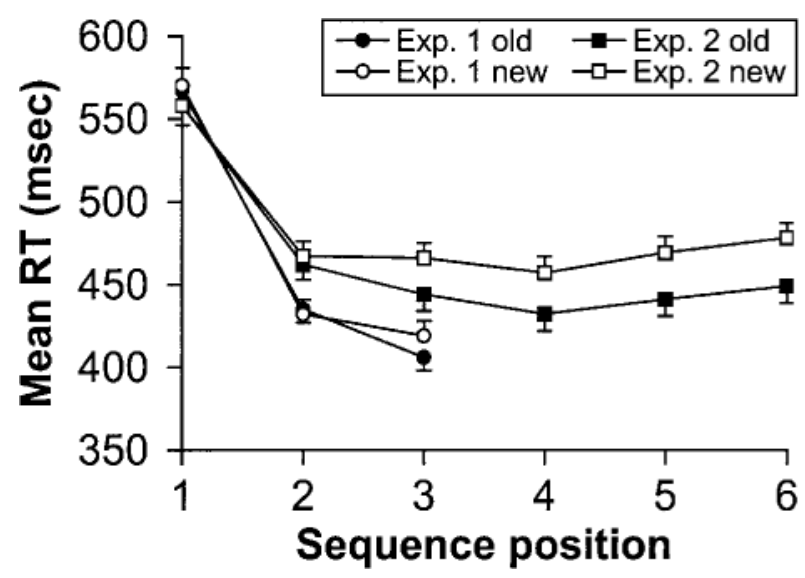

Figure 2. Mean ( $\pm S E M$ ) reaction time to Targets 1-3 of old and new test sequences in Experiment 1 and Targets 1-6 in Experiment 2. Targets 3-6 are predictable from the preceding targets, whereas targets 1-2 are not predictable. 


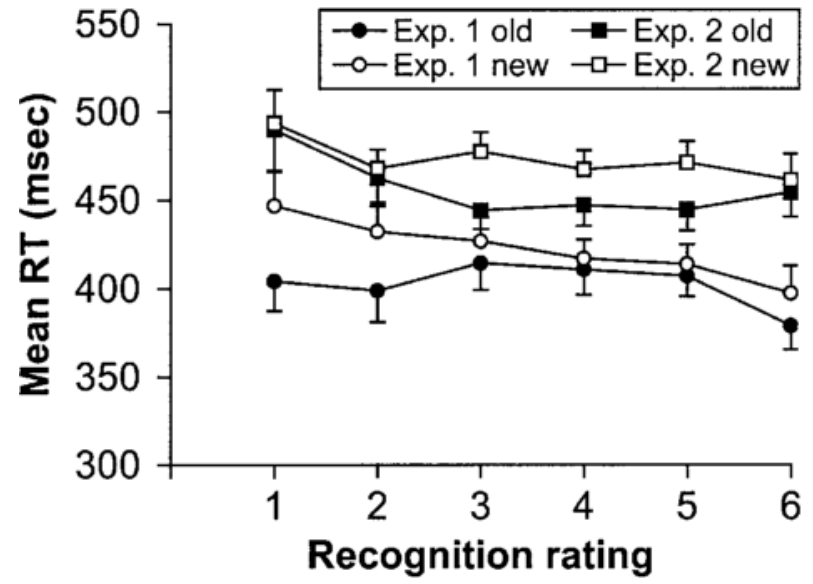

Figure 3. Mean ( $\pm S E M)$ response latency (in milliseconds) to targets in old and new test sequences is plotted on the ordinate as a function of recognition rating $(1=$ certain $n e w, 6=$ certain old $)$ on the abscissa. Data are based on RTs to Target 3 of each sequence in Experiment 1 and on mean RTs across Targets 3-6 of each sequence in Experiment 2. Targets 1-2 were unpredictable in both old and new sequences.

ure 3 presents RTs for old and new sequences given ratings of $1,2, \ldots, 6$, again computed excluding the first and second targets of each test sequence. It is plain that the predictable targets in the old sequences elicited more rapid responses than the corresponding unpredictable targets in the new sequences at each recognition score. Combined across recognition ratings, the old/new difference is significant in both experiments $[t(68)=2.90, p<.005$ in Experiment 1 , and $t(78)=6.43, p<.001$ in Experiment 2]. Paired comparisons revealed that in Experiment 1, RTs on old versus new sequences differed for ratings of 1 and 2 $[t(19)=2.73, p<.02$ and $t(36)=1.74, p<.05$, respectively], but not for ratings of $3-6[t<1.42, d f=38$ to 63 , $p>.15$ in each case]. In Experiment 2, RTs differed for ratings of 3,4 , and $5[t(57)=5.15, p<.001, t(59)=3.66$, $p<.001$, and $t(67)=4.19, p<.001$, respectively], but not for ratings of 1,2 , or $6[t<0.86, d f=27$ to $53, p>.3$ in each case]. Thus the direct (recognition) and indirect (priming) measures are dissociable: Even when old and new test sequences received identical recognition ratings, old sequences were nevertheless executed more rapidly than were new ones.

This effect is also quite consistent across participants: Averaging across recognition ratings, $47 / 69$ (68\%) of participants in Experiment 1 and 63/79 (80\%) of those in Experiment 2 had shorter response latencies overall to old than to new sequences which they did not discriminate in recognition. These results therefore demonstrate response priming of old relative to new sequences that is not accompanied by differential recognition. Indeed, the priming effect is significant in Experiment 1 for sequences that were not recognized at all (i.e., sequences given a rating of 1).

Note that the effect was not due to a selection artifact, for instance, some test sequences simply being executed faster than others because of their precise finger move- ment properties. Although some response sequences were indeed executed faster than others, the old/new effect was significant when computed across all test items. Counterbalancing ensures that unconditional execution speed differences should cancel out between old and new test sequences.

The old/new difference is largest at the endpoints of the rating scale in Experiment 1 and around the midpoint of the scale in Experiment 2. Whether anything should be read into this pattern must remain a question for future studies. The model presented below sometimes yields patterns similar to those in Experiment 1 and sometimes similar to those in Experiment 2, but these variations are merely due to sampling error.

\section{DISCUSSION}

These experiments explored the relationship between chronometric and recognition measures of sequence knowledge and revealed compelling evidence that these measures can be dissociated. Even when old and new test sequences received identical recognition ratings, old sequences were executed more rapidly than were new ones. This is the first demonstration of implicit priming of a sequentially structured response chain under conditions in which priming and recognition were assessed concurrently. Previous research (Cleeremans \& McClelland, 1991; Destrebecqz \& Cleeremans, 2001; Frensch et al., 1998; Honda et al., 1998; Jiménez et al., 1996; Nissen \& Bullemer, 1987; Perruchet \& Amorim, 1992; Perruchet et al., 1997; Reber \& Squire, 1998; Reed \& Johnson, 1994; Stadler, 1995; Willingham et al., 1993) has not allied contiguous measurement on direct and indirect tests with the analytic procedure of examining performance on the indirect test at different levels of performance on the direct test, which means that fine-grained comparison of measures across test items has not been possible. The present study shows that such fine-grained comparison is essential, since global measures of priming and recognition were strongly associated (see Figure 1).

The present findings provide an analogue in sequence learning to effects reported in implicit memory for words showing differential event-related brain potentials in parietal areas to unrecognized old versus new words (Rugg et al., 1998). Such words, despite not being differentially recognized, yield different signatures of brain activity interpreted as reflecting the neural localization of implicit memory.

The interpretation of dissociations between direct and indirect tests of sequence knowledge is complex. One view is that such dissociations reflect the influence of independent neural and functional systems, with direct tests being dependent on a declarative memory system in the medial temporal lobes and indirect tests being controlled by other brain systems (Reber \& Squire, 1998). In the case of implicit perceptual-motor sequence learning, it is likely that brain circuits including primary sensorimotor cortex (Honda et al., 1998) and premotor, striatal, and thalamic regions (Curran, 1998; Maquet et al., 2000) are critical for prim- 


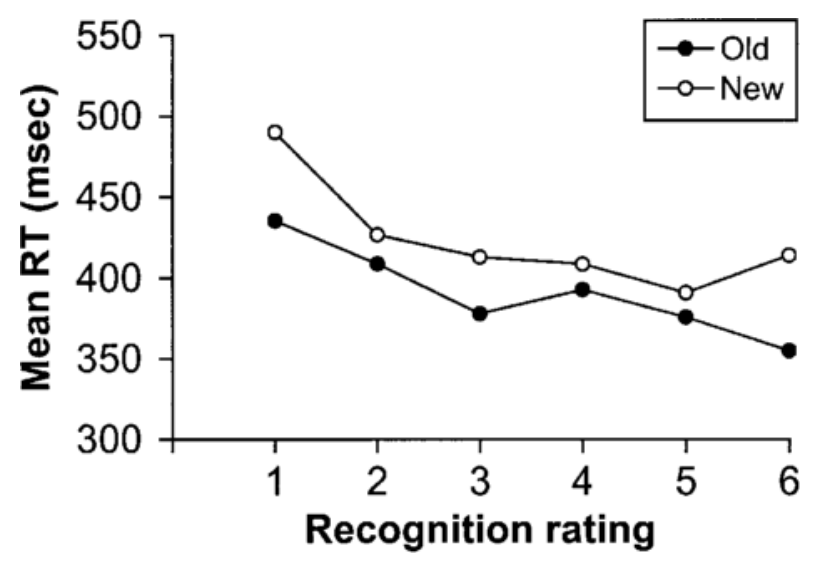

Figure 4. Mean simulated reaction times (in milliseconds) to targets in old and new test sequences as a function of recognition rating.

ing, though brain imaging studies have yet to use sensitive methods, such as those developed here, to dissociate implicit and explicit measures of sequence knowledge and hence isolate the relevant priming effects.

An alternative possibility, however, is that a single knowledge source underlies performance on both types of test, with subtle differences between the retrieval processes recruited by the tests being responsible for the observed dissociations (Kinder \& Shanks, 2001; Nosofsky \& Zaki, 1998, 1999). We next present such a model that reveals that priming can be dissociated from recognition in the manner found in the present experiments, even if the two measures depend on the same underlying memory variable.

\section{A Model of Priming and Recognition}

Our model, which is conceptually very similar to standard signal detection theory models for recognition judgments and their latencies (Pike, 1973; Ratcliff \& Murdock, 1976), starts with the simple assumption that new and old test items are associated with a memory strength variable that we will call familiarity $f$. Greater degrees of familiarity lead to higher recognition judgments and faster RTs, and familiarity can be thought of as some composite, but unidimensional, function of the perceptual familiarity of the stimulus sequence and the motor fluency of the executed response sequence. In the model, $f$ is a uniformly distributed random variable in the interval $[0, .8]$ for new items and in the interval $[.2,1]$ for old items. ${ }^{3}$ Thus the mean familiarity of old items, $f_{\text {old }}$, is slightly higher (by .2) than the mean for new items, $f_{\text {new }}$. For each participant, a single value of familiarity is independently sampled for new and old items from these distributions. Next, we assume that RT is a decreasing function of $f$ but with the addition of some random error:

$$
\begin{aligned}
& \mathrm{RT}_{\text {old }}=200+100\left(1-f_{\text {old }}\right)+300 e, \\
& \mathrm{RT}_{\text {new }}=200+100\left(1-f_{\text {new }}\right)+300 e,
\end{aligned}
$$

where $e$ is uniformly distributed random error in the interval $[0,1]$. These equations generate RTs between a max- imum of $600 \mathrm{msec}$ when the familiarity of the test item is zero and a minimum of $200 \mathrm{msec}$ when familiarity is 1 .

Recognition judgments $(J)$ are also based on familiarity, but include another (independent) source of error:

$$
\begin{aligned}
& J_{\text {old }}=2 f_{\text {old }}+3 e+1, \\
& J_{\text {new }}=2 f_{\text {new }}+3 e+1,
\end{aligned}
$$

where $e$ is again uniformly distributed random error in the interval $[0,1] . J$ is rounded to the nearest integer value. These equations generate recognition ratings between a maximum of $600 \mathrm{msec}$ when the familiarity of the test item approaches 1 and a minimum of 1 when familiarity is 0 .

Despite the fact that RTs and recognitionjudgments depend on the same variable $(f)$ in this model, and depend on nothing else apart from noise, the model nevertheless generates a pattern of data strikingly similar to that shown in Figure 3. Figure 4 presents the mean RTs to old and new items at each recognition judgment based on 1,000 simulated subjects. RTs are faster to old items than to new items simply as an automatic by-product of the fact that the two measures are affected by the random variation and measurement error that plague any experimental measure. More specifically, for old and new sequences to be rated equally in recognition, a larger value of $e$ in Equation 4 compared with Equation 3 is necessary, on average, to offset the larger average value of $f_{\text {old }}$ compared with $f_{\text {new }}$. However, when these same $f$ values are used to determine RTs in Equations 1 and 2, they will be combined with independently generated values of $e$. Since the latter are uncorrelated with the $e$ values incorporated in the recognition judgments, on average they will not differ for old and new items. Hence, since $f_{\text {old }}$ is on average greater than $f_{\text {new }}$, $\mathrm{RT}_{\text {old }}$ will be lower than $\mathrm{RT}_{\text {new }}$, as observed empirically in the participants' behavior.

When error is not included in the model, the old-new difference is zero. This confirms that it is the imperfect relationship between measures of priming and recognition that is responsible for the effect.

\section{Summary}

The present work supports an empirical conclusion and a theoretical one. Empirically, practiced sequences of responses are executed faster than unpracticed ones, even when the sequences are given identical recognition ratings. This is a clear confirmation that priming and recognition can be dissociated. However, the theoretical conclusion is that this dissociation is to be expected from any pair of measures that are less than perfectly correlated and is not inconsistent with a model in which priming and recognition depend on the same underlying memory trace.

\section{REFERENCES}

Cleeremans, A., \& McClelland, J. L. (1991). Learning the structure of event sequences. Journal of Experimental Psychology: General, 120, 235-253.

Cohen, A., Ivry, R. I., \& Keele, S. W. (1990). Attention and structure in sequence learning. Journal of Experimental Psychology: Learning, Memory, \& Cognition, 16, 17-30. 
Cohen, N. J., \& Eichenbaum, H. (1993). Memory, amnesia, and the hippocampal system. Cambridge, MA: MIT Press.

Curran, T. (1997). Higher-order associative learning in amnesia: Evidence from the serial reaction time task. Journal of Cognitive Neuroscience, 9, 522-533.

Curran, T. (1998). Implicit sequence learning from a cognitive neuroscience perspective: What, how, and where? In M. A. Stadler \& P. A. Frensch (Eds.), Handbook of implicit learning (pp. 365-400). Thousand Oaks, CA: Sage.

Destrebecqz, A., \& Cleeremans, A. (2001). Can sequence learning be implicit? New evidence with the process dissociation procedure. Psychonomic Bulletin \& Review, 8, 343-350.

Dienes, Z, \& BERry, D. (1997). Implicit learning: Below the subjective threshold. Psychonomic Bulletin \& Review, 4, 3-23.

Fendrich, D. W., HeAly, A. F., \& Bourne, L. E. (1991). Long-term repetition effects for motoric and perceptual procedures. Journal of Experimental Psychology: Learning, Memory, \& Cognition, 17, 137-151.

Frensch, P. A., Lin, J., \& Buchner, A. (1998). Learning versus behavioral expression of the learned: The effects of a secondary tonecounting task on implicit learning in the serial reaction task. Psychological Research, 61, 83-98.

Gabrieli, J. D. E. (1998). Cognitive neuroscience of human memory. Annual Review of Psychology, 49, 87-115.

Honda, M., Deiber, M.-P., IbáÑez, V., Pascual-Leone, A., Zhuang, P., \& Hallett, M. (1998). Dynamic cortical involvement in implicit and explicit motor sequence learning: A PET study. Brain, 121, 2159-2173.

Jiménez, L., Méndez, C., \& Cleeremans, A. (1996). Comparing direct and indirect measures of sequence learning. Journal of Experimental Psychology: Learning, Memory, \& Cognition, 22, 948-969.

Kinder, A., \& Shanks, D. R. (2001). Amnesia and the declarative/nondeclarative distinction: A recurrent network model of classification, recognition, and repetition priming. Journal of Cognitive Neuroscience, 13, 648-669.

Maquet, P., Laureys, S., Peigneux, P., Fuchs, S., Petiau, C., Phillips, C., Aerts, J., Del Fiore, G., Degueldre, C., Meulemans, T., Luxen, A., Franck, G., Van Der Linden, M., Smith, C., \& Cleeremans, A. (2000). Experience-dependent changes in cerebral activation during human REM sleep. Nature Neuroscience, $\mathbf{3}$, 831-836.

MarsoleK, C. J., \& Field, J. E. (1999). Perceptual-motor sequence learning of general regularities and specific sequences. Journal of Experimental Psychology: Human Perception \& Performance, 25, 815-836.

Nissen, M. J., \& Bullemer, P. (1987). Attentional requirements of learning: Evidence from performance measures. Cognitive Psychology, 19, 1-32.

NosOFSKY, R. M., \& ZAKI, S. R. (1998). Dissociations between categorization and recognition in amnesic and normal individuals: An exemplar-based interpretation. Psychological Science, 9, 247-255.

NosofSKY, R. [M.], \& ZAKI, S. [R.] (1999). Math modeling, neuropsychology, and category learning: Response to B. Knowlton (1999). Trends in Cognitive Sciences, 3, 125-126.

Perruchet, P., \& Amorim, M.-A. (1992). Conscious knowledge and changes in performance in sequence learning: Evidence against dissociation. Journal of Experimental Psychology: Learning, Memory, \& Cognition, 18, 785-800.

Perruchet, P., Bigand, E., \& Benoit-Gonin, F. (1997). The emergence of explicit knowledge during the early phase of learning in sequential reaction time tasks. Psychological Research, 60, 4-13.

Perruchet, P., \& Gallego, J. (1993). Association between conscious knowledge and performance in normal subjects: Reply to Cohen and Curran (1993) and Willingham, Greeley, and Bardone (1993). Journal of Experimental Psychology: Learning, Memory, \& Cognition, 19, 1438-1444.

Perruchet, P., Gallego, J., \& Savy, I. (1990). A critical reappraisal of the evidence for unconscious abstraction of deterministic rules in complex experimental situations. Cognitive Psychology, 22, 493-516.

PIKE, R. (1973). Response latency models for signal detection. Psychological Review, 80, 53-68.

RAtcliff, R, \& Murdock, B. B. (1976). Retrieval processes in recognition memory. Psychological Review, 83, 190-214.

Reber, P. J., \& SQUIRE, L. R. (1998). Encapsulation of implicit and explicit memory in sequence learning. Journal of Cognitive Neuroscience, $\mathbf{1 0}$, 248-263.

ReEd, J., \& Johnson, P. (1994). Assessing implicit learning with indirect tests: Determining what is learned about sequence structure. Journal of Experimental Psychology: Learning, Memory, \& Cognition, 20, 585-594.

Rugg, M. D., Mark, R. E., Walla, P., Schloerscheidt, A. M., Birch, C. S., \& Allan, K. (1998). Dissociation of the neural correlates of implicit and explicit memory. Nature, 392, 595-598.

Schacter, D. L., \& Buckner, R. L. (1998). Priming and the brain. Neuron, 20, 185-195.

Shanks, D. R, \& Johnstone, T. (1998). Implicit knowledge in sequential learning tasks. In M. A. Stadler \& P. A. Frensch (Eds.), Handbook of implicit learning (pp. 533-572). Thousand Oaks, CA: Sage.

Shanks, D. R, \& Johnstone, T. (1999). Evaluating the relationship between explicit and implicit knowledge in a sequential reaction time task. Journal of Experimental Psychology: Learning, Memory, \& Cognition, 25, 1435-1451.

Shanks, D. R., \& St. John, M. F. (1994). Characteristics of dissociable human learning systems. Behavioral \& Brain Sciences, 17, 367-447.

STADLER, M. A. (1995). Role of attention in sequence learning. Journal of Experimental Psychology: Learning, Memory, \& Cognition, 21, 674-685.

Willingham, D. B., Greeley, T., \& Bardone, A. M. (1993). Dissociation in a serial response time task using a recognition measure: Comment on Perruchet and Amorim 1992. Journal of Experimental Psychology: Learning, Memory, \& Cognition, 19, 1424-1430.

\section{NOTES}

1. Reaction times increased in each case on this transfer block, providing confirmation that sequence learning was occurring during the training stage. However, data from the training phase are of little interest here since all they serve to show is that the participants learned about the sequences, which is independently demonstrated in the test results. Hence, training-stage data are not reported.

2. Although this is a cross-experiment comparison and the participants were not randomly allocated to the experiments, in all other respects, the experiments were run identically (e.g., same computers and software, testing environment, instructions, etc.).

3. The qualitative predictions of the model are identical if familiarity is normally distributed. The model is conceptually slightly simpler if uniform distributions are used.

(Manuscript received September 21, 2000; revision accepted for publication March 4, 2001.) 